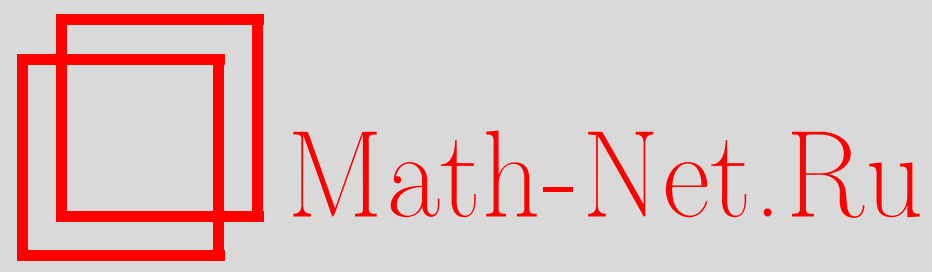

А. С. Леонов, Об $H$-свойстве функционалов в пространствах Соболева, Матем. заметки, 2005, том 77, выпуск $3,378-394$

DOI: https://doi.org/10.4213/mzm2500

Использование Общероссийского математического портала Math-Net.Ru подразумевает, что вы прочитали и согласны с пользовательским соглашением http://www.mathnet.ru/rus/agreement

Параметры загрузки:

IP: 52.6 .47 .48

26 апреля 2023 г., 04:15:03

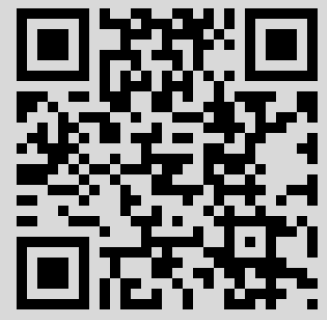




\section{ОБ $Н$-СВОЙСТВЕ ФУНКЦИОНАЛОВ В ПРОСТРАНСТВАХ СОБОЛЕВА}

\section{А. С. Леонов}

В статье рассматриваются специальные классы усиленно выпуклых функционалов в пространствах Соболева. Доказывается, что функционалы из таких классов обладают так называемьм $H$-свойством: для них из слабой сходимости последовательностей аргументов и сходимости этих последовательностей по функционалу вытекает сильная сходимость.

Библиография: 8 названий.

1. Введение. Пусть $Z$ - банахово пространство с элементами $z$, а $D, D \subset Z$,некоторое непустое множество. Предположим, что на $D$ определен функционал $\Omega(z)$.

ОПРЕДЕЛЕНИЕ 1.1. Функционал $\Omega(z)$ имеет на множестве $D H$-свойство, если для любой последовательности $\left\{z_{n}\right\} \subset D$ из ее слабой сходимости при $n \rightarrow \infty$ к некоторому пределу $z_{0} \in D, z_{n} \rightarrow z_{0}$, и ее сходимости по функционалу (т.е. сходимости $\left.\Omega\left(z_{n}\right) \rightarrow \Omega\left(z_{0}\right)\right)$ следует сильная сходимость $\left(z_{n} \rightarrow z_{0}\right)$.

Типичным примером функционала с $H$-свойством служит норма в гильбертовом пространстве, а также нормы в “пространствах с $H$-свойством" (см., например, [1]), в которых сходимости $z_{n} \rightarrow z_{0},\left\|z_{n}\right\| \rightarrow\left\|z_{0}\right\|$ по определению порождают сильную сходимость $z_{n} \rightarrow z_{0}$ для каждой $\left\{z_{n}\right\} \subset Z$. В частности, $H$-свойство имеют пространства Лебега $L_{p}, p>1$, и Соболева $W_{p}^{l}, p>1, l \geqslant 1,[2]$ и их нормы. Примеры функционалов с $H$-свойством в пространстве $L_{1}$ представлены в [3]. Из результатов этой работы (теоремы 2.5 и 3.2) следует, что интегральные функционалы вида

$$
\Omega(z)=\int_{0}^{1} f[z(x)] d x
$$

обладают $H$-свойством на множестве $D_{+}=\{z(x) \geqslant 0, \Omega(z)<\infty\}$ пространства $L_{1}[0,1]$, если $f(z) \in C[0,+\infty) \cap C^{2}(0,+\infty)$, производная $f^{\prime \prime}(z)>0$ монотонна при $z>0$ и найдется такое число $\rho, 0 \leqslant \rho<1$, что $f^{\prime \prime}(z) z^{\rho} \geqslant \sigma=$ const $>0$ при всех $z>0$. Можно привести и примеры функционалов, которые не имеют $H$-свойства. Так, в пространстве $L_{2}[0,1]$ интегральньй функционал вида $(1.1)$ с $f(z)=|z|^{2} /(1+|z|)$ не имеет $H$-свойства на всем пространстве. В этом легко убедиться, рассмотрев последовательность

$$
z_{n}(x)= \begin{cases}\sqrt{n} \sin (n), & 0 \leqslant x \leqslant 1 / n \\ 0, & 1 / n<x \leqslant 1 .\end{cases}
$$

Работа выполнена при финансовой поддержке Российского фонда фундаментальных исследований, грант № 02-01-00044. 
Эта последовательность слабо сходится в $L_{2}[0,1]$ к нулю и, как можно вычислить, сходится по функционалу (1.1): $\Omega\left(z_{n}\right) \rightarrow \Omega(0)$. Тем не менее, сильно к нулю она не сходится, так как $\left\|z_{n}-0\right\|_{L_{2}}^{2}=\sin ^{2}(n)$. Заметим, что в этом примере $\Omega(z) \leqslant\|z\|_{L_{2}}^{2}$. Можно также привести пример функционала (1.1), удовлетворяюшего неравенствам $k\|z\|_{L_{2}}^{2} \leqslant \Omega(z) \leqslant\|z\|_{L_{2}}^{2}, k=$ const $>0$, но не имеющего $H$-свойства в $L_{2}[0,1]$.

В связи с этим возникает вопрос: какие еще функционалы, кроме перечисленных, обладают $H$-свойством и как описать классы этих функционалов в конкретных пространствах (например, в пространствах Соболева). Этому и посвящена данная работа.

\section{2. Усиленно выпуклые функционалы.}

ОПРЕДЕЛЕНИЕ 2.1. Функционал $\Omega(z)$ назьвается усиленно выпуклым на выпуклом множестве $D, D \subset Z$, если для него сушествует определенньй на $D \times D$ функционал $\Delta\left(z_{1}, z_{2}\right) \geqslant 0$, тождественно не равньй нулю, такой, что

$$
\frac{\Omega\left(z_{1}\right)+\Omega\left(z_{2}\right)}{2} \geqslant \Omega\left(\frac{z_{1}+z_{2}}{2}\right)+\Delta\left(z_{1}, z_{2}\right) \quad \forall z_{1}, z_{2} \in D .
$$

Заметим, что, если условие (2.1) выполнено с $\Delta\left(z_{1}, z_{2}\right)=\xi\left(\left\|z_{1}-z_{2}\right\|\right)$, где $\xi(t)$ - непрерьвная и монотонно возрастающая при $t \geqslant 0$ функция, $\xi(0)=0$, то такой функционал $\Omega$ назьвается (строго) равномерно выпуклым на $D$ (см., например, [4]).

ОПРЕДЕЛЕНИЕ 2.2. Будем говорить, что функционал $\Delta\left(z_{1}, z_{2}\right)$ имеет на множестве $D S$-свойство, если для любой последовательности $\left\{z_{n}\right\} \subset D$ из сходимостей $z_{n} \rightarrow$ $z_{0} \in D, \Delta\left(z_{n}, z_{0}\right) \rightarrow 0$ при $n \rightarrow \infty$ следует сильная сходимость $z_{n} \rightarrow z_{0}$.

ЛЕмма 2.3. Предположим, что усиленно выпуклый на выпуклом множестве $D$ функиионал $\Omega(z)$ слабо полунепрерывен снизу на этом множестве и соответствующий функиионал $\Delta\left(z_{1}, z_{2}\right)$ из определения 2.1 имеет $S$-свойство. Тогда функиионал $\Omega(z)$ обладает $H$-свойством на $D$.

ДоКАЗАТЕЛЬСТво. Пусть произвольная последовательность $\left\{z_{n}\right\} \subset D$ слабо сходится к элементу $z_{0} \in D$ и сходится к этому элементу по функционалу $\Omega$. Тогда по определению слабой полунепрерьвности снизу функционала $\Omega(z)$ получим предельное соотношение:

$$
\liminf _{n \rightarrow \infty} \Omega\left(\frac{z_{n}+z_{0}}{2}\right) \geqslant \Omega\left(z_{0}\right) .
$$

Применяя его вместе со сходимостью по функционалу: $\Omega\left(z_{n}\right) \rightarrow \Omega\left(z_{0}\right)$ в неравенстве (2.1), написанном для $z_{1}=z_{n}, z_{2}=z_{0}$, получим

$$
\begin{aligned}
\Omega\left(z_{0}\right) & =\lim _{n \rightarrow \infty}\left[\frac{\Omega\left(z_{n}\right)+\Omega\left(z_{0}\right)}{2}\right] \geqslant \lim _{n \rightarrow \infty}\left[\Omega\left(\frac{z_{n}+z_{0}}{2}\right)+\Delta\left(z_{n}, z_{0}\right)\right] \\
& \geqslant \liminf _{n \rightarrow \infty} \Omega\left(\frac{z_{n}+z_{0}}{2}\right)+\lim _{n \rightarrow \infty} \Delta\left(z_{n}, z_{0}\right) \geqslant \Omega\left(z_{0}\right)+\lim _{n \rightarrow \infty} \Delta\left(z_{n}, z_{0}\right) .
\end{aligned}
$$

Отсюда следует сходимость $\Delta\left(z_{n}, z_{0}\right) \rightarrow 0$. Из нее и из $S$-свойства функционала $\Delta$ получается сильная сходимость $z_{n} \rightarrow z_{0}$. Это доказьвает, что функционал $\Omega$ имеет $H$-свойство.

Результат леммы 2.3 верен, например, для слабо полунепрерывных снизу и строго равномерно выпуклых функционалов, так как соответствующий функционал $\Delta\left(z_{1}, z_{2}\right)=$ $\xi\left(\left\|z_{1}-z_{2}\right\|\right)$ очевидно имеет $S$-свойство. Другие примеры приводятся ниже. 
3. Усиленно выпуклые функционалы с $H$-свойством в пространствах Соболева. Везде далее будем считать, что $Z$ - это пространство Соболева $W_{p}^{l}(T), p>1$, $l \geqslant 1$, функций $z(x)$, определенных в замкнутой ограниченной области $T \subset \mathbb{R}^{N}$ с достаточно гладкой гранищей (см. [2]), норма в котором задается равенством

$$
\|z\|=\|z\|_{W_{p}^{l}}=\left\{\sum_{0 \leqslant|\alpha| \leqslant l} \int_{T}\left|D^{\alpha} z(x)\right|^{p} d x\right\}^{1 / p}=\left\{\sum_{0 \leqslant|\alpha| \leqslant l}\left\|D^{\alpha} z\right\|_{p}^{p}\right\}^{1 / p} .
$$

Здесь

$$
D^{\alpha} z(x)=\frac{\partial^{|\alpha|} z}{\partial x_{1}^{\alpha_{1}} \cdots \partial x_{N}^{\alpha_{N}}}, \quad|\alpha|=\alpha_{1}+\cdots+\alpha_{N}, \quad \alpha_{1}, \ldots, \alpha_{N}=0,1, \ldots,
$$

a

$$
\|z\|_{p}=\left\{\int_{T}|z(x)|^{p} d x\right\}^{1 / p}
$$

- норма в пространстве $L_{p}(T)$. Число слагаемых в сумме (3.1) будем в дальнейшем обозначать как $\nu$.

Предположим, что заданы линейные операторы вида

$$
A_{0} z(x)=z(x), \quad A_{k} z(x)=\sum_{0 \leqslant|\alpha| \leqslant l} a_{k \alpha} D^{\alpha} z(x), \quad k=1, \ldots, m,
$$

с непрерьвньми в $T$ коэффициентами $a_{k \alpha}=a_{k \alpha}(x)$. При этом считаем вьполненным условие

$$
\sum_{k=0}^{m}\left\|A_{k} z\right\|_{p}^{p} \geqslant c_{0}\|z\|_{W_{p}^{l}}^{p}, \quad c_{0}=\text { const }>0, \quad z \in W_{p}^{l}(T) .
$$

Рассмотрим сначала функционалы специального вида, определенные на заданном выпуклом множестве $D \subset W_{p}^{l}(T)$ и имеющие форму

$$
\bar{\Omega}(z)=\bar{\Omega}[z(x)]=\int_{T} f\left(x,\left|A_{0} z(x)\right|,\left|A_{1} z(x)\right|, \ldots,\left|A_{m} z(x)\right|\right) d x, \quad z(x) \in D .
$$

В дальнейшем будем предполагать, что производящая функция $f(x, y)=f\left(x, y_{0}, y_{1}, \ldots\right.$, $\left.y_{m}\right)$ этого функционала имеет следующие свойства:

а) она определена и непрерывна при $x \in T, y=\left(y_{0}, y_{1}, \ldots, y_{m}\right) \in \mathbb{R}_{+}^{m+1}=\left\{y_{0} \geqslant 0\right.$, $\left.y_{1} \geqslant 0, \ldots, y_{m} \geqslant 0\right\}$

б) она не убывает по каждой переменной $y_{k}, k=0, \ldots, m$, при фиксированных других переменных, имеющих допустимые значения;

в) она выпукла по $y$ в $\mathbb{R}_{+}^{m+1}$ при каждом $x \in T$.

Заметим, что из а)-в) следует ограниченность этой функции снизу. Поэтому без ущерба для общности будем считать, что $f(x, y) \geqslant 0$.

Лемма 3.1. Если производящая функиия $f(x, y)$ имеет свойства а)-в), то функиионал (3.3) слабо полунепрерывен снизу на выпуклом мнохестве $D \subset W_{p}^{l}(T)$. 
ДокАЗАТЕльСтво. Прежде всего, отметим, что функция $f(x,|y|)=f\left(x,\left|y_{0}\right|,\left|y_{1}\right|\right.$, $\left.\ldots,\left|y_{m}\right|\right)$ в силу условий а)-в) будет непрерывной и выпуклой по $y$ в $\mathbb{R}^{m+1}$ при каждом $x \in T$. Поэтому на множестве $D$ выполнено условие выпуклости функционала (3.3)

$$
\begin{aligned}
\frac{\bar{\Omega}\left(z_{1}\right)+\bar{\Omega}\left(z_{2}\right)}{2}= & \int_{T}\left(\frac{f\left(x,\left|z_{1}(x)\right|,\left|A_{1} z_{1}(x)\right|, \ldots,\left|A_{m} z_{1}(x)\right|\right)}{2}\right. \\
& \left.+\frac{f\left(x,\left|z_{2}(x)\right|,\left|A_{1} z_{2}(x)\right|, \ldots,\left|A_{m} z_{2}(x)\right|\right)}{2}\right) d x \\
\geqslant & \int_{T} f\left(x,\left|\frac{z_{1}(x)+z_{2}(x)}{2}\right|,\left|A_{1}\left(\frac{z_{1}(x)+z_{2}(x)}{2}\right)\right|, \ldots,\right. \\
= & \bar{\Omega}\left(\frac{z_{1}+z_{2}}{2}\right) \quad \forall z_{1}, z_{2} \in D
\end{aligned}
$$

и, кроме того, $\bar{\Omega}(z) \geqslant 0$. Докажем теперь сильную полунепрерьвность снизу этого функционала на множестве $D$ пространства $W_{p}^{l}(T)$. Возьмем любую последовательность $\left\{z_{n}\right\} \subset D$ такую, что $z_{n} \rightarrow z_{0} \in D$ при $n \rightarrow \infty$. Если при этом

$$
\liminf _{n \rightarrow \infty} \bar{\Omega}\left(z_{n}\right)=+\infty
$$

то

$$
\liminf _{n \rightarrow \infty} \bar{\Omega}\left(z_{n}\right) \geqslant \bar{\Omega}\left(z_{0}\right)
$$

и условие сильной полунепрерьвности снизу вьполнено. Если же предел

$$
s=\liminf _{n \rightarrow \infty} \bar{\Omega}\left(z_{n}\right)
$$

конечен, то найдется такая подпоследовательность $\left\{z_{n_{i}}\right\} \subset\left\{z_{n}\right\}$, для которой

$$
\lim _{i \rightarrow \infty} \bar{\Omega}\left(z_{n_{i}}\right)=s
$$

и, кроме того, $z_{n_{i}} \rightarrow z_{0}, D^{\alpha} z_{n_{i}} \rightarrow D^{\alpha} z_{0} \forall \alpha,|\alpha|=1, \ldots, l$, почти всюду в $T$. Последние две сходимости можно обеспечить, так как из условия $\left\|z_{n}-z_{0}\right\|_{W_{p}^{l}} \rightarrow 0$ следует, что $D^{\alpha} z_{n} \rightarrow D^{\alpha} z_{0}$ сильно в $L_{p}(T)$ при каждом $\alpha,|\alpha|=0,1, \ldots, l$. Таким образом, почти всюду в $T$ имеется сходимость

$$
f\left(x,\left|z_{n_{i}}(x)\right|,\left|A_{1} z_{n_{i}}(x)\right|, \ldots,\left|A_{m} z_{n_{i}}(x)\right|\right) \rightarrow f\left(x,\left|z_{0}(x)\right|,\left|A_{1} z_{0}(x)\right|, \ldots,\left|A_{m} z_{0}(x)\right|\right) .
$$

Отсюда и из ограниченности последовательности $\left\{\bar{\Omega}\left(z_{n_{i}}\right)\right\}$ по лемме Фату получим

$$
\begin{aligned}
\bar{\Omega}\left(z_{0}\right) & =\int_{T} f\left(x,\left|z_{0}(x)\right|,\left|A_{1} z_{0}(x)\right|, \ldots,\left|A_{m} z_{0}(x)\right|\right) d x \\
& \leqslant \liminf _{i \rightarrow \infty} \int_{T} f\left(x,\left|z_{n_{i}}(x)\right|,\left|A_{1} z_{n_{i}}(x)\right|, \ldots,\left|A_{m} z_{n_{i}}(x)\right|\right) d x \\
& =\lim _{i \rightarrow \infty} \bar{\Omega}\left(z_{n_{i}}\right)=\liminf _{i \rightarrow \infty} \bar{\Omega}\left(z_{n}\right),
\end{aligned}
$$

т.е. и в этом случае вьполнено условие сильной полунепрерьвности снизу функционала (3.3). В заключение заметим, что слабая полунепрерьвность снизу этого функционала вытекает из его сильной полунепрерывности снизу и выпуклости на $D[4]$. 
ТЕОрема 3.2. Предположим, что производящая функиия $f(x, y)$ функционала (3.3) обладает свойствами а), б) и, кроме того, усиленно выпукла в следующем смысле:

$$
\begin{gathered}
\frac{f(x,|u|)+f(x,|v|)}{2} \geqslant f\left(x,\left|\frac{u+v}{2}\right|\right)+\kappa \sum_{k=0}^{m}|| u_{k}|-| v_{k}||^{p} \\
\forall x \in T, \quad u, v \in \mathbb{R}^{m+1}, \quad \kappa=\text { const }>0 .
\end{gathered}
$$

Тогда соответствующий функиионал (3.3) имеет $H$-свойство на мнохестве $D$.

ДокАЗАтЕльСтво. Для функционала (3.3) из условия (3.5) с $u_{k}=A_{k} z_{1}(x), v_{k}=$ $A_{k} z_{2}(x) \forall z_{1}, z_{2} \in D$ по аналогии с (3.4) получается оценка (2.1), в которой

$$
\Delta\left(z_{1}, z_{2}\right)=\left.\kappa \sum_{k=0}^{m} \int_{T}|| A_{k} z_{1}(x)|-| A_{k} z_{2}(x)\right|^{p} d x
$$

Поэтому функционал (3.3) оказьвается усиленно выпуклым. Покажем, что такой функционал $\Delta\left(z_{1}, z_{2}\right)$ имеет на $D S$-свойство. Возьмем любую последовательность $\left\{z_{n}\right\} \subset D$, для которой

$$
z_{n} \stackrel{W_{p}^{l}}{\longrightarrow} z_{0} \in D \text { и } \Delta\left(z_{n}, z_{0}\right) \rightarrow 0 \quad \text { при } n \rightarrow \infty .
$$

Тогда из вида функционала $\Delta$ следует

$$
\left\|A_{k} z_{n}\right\|_{p} \rightarrow\left\|A_{k} z_{0}\right\|_{p} \quad \forall k
$$

Из слабой сходимости $z_{n} \rightarrow z_{0}$ в $W_{p}^{l}$ вытекают слабые сходимости

$$
D^{\alpha} z_{n} \stackrel{L_{p}}{\longrightarrow} D^{\alpha} z_{0} \quad \forall \alpha, \quad 0 \leqslant|\alpha| \leqslant l,
$$

и, далее, $A_{k} z_{n} \stackrel{L_{p}}{\longrightarrow} A_{k} z_{0}$. Тогда из $H$-свойства пространства $L_{p}$ следует соотношение $A_{k} z_{n} \stackrel{L_{p}}{\rightarrow} A_{k} z_{0}$. Оно вместе с вытекающей из условия (3.2) оценкой

$$
\sum_{k=0}^{m}\left\|A_{k} z_{n}-A_{k} z_{0}\right\|_{p}^{p} \geqslant c_{0}\left\|z_{n}-z_{0}\right\|_{W_{p}^{l}}^{p}
$$

приводит к сильной сходимости $z_{n} \rightarrow z_{0}$ в $W_{p}^{l}$. Таким образом, получилось, что из сходимостей $z_{n} \rightarrow z_{0}, \Delta\left(z_{n}, z_{0}\right) \rightarrow 0$ следует сильная сходимость $z_{n} \rightarrow z_{0}$. Это означает выполнение $S$-свойства для $\Delta\left(z_{1}, z_{2}\right)$. Так как по условиям теоремы производящая функция $f(x, y)$ имеет свойства а), б) и вытекающее из (3.5) свойство в), то по лемме 3.1 усиленно вьпуклый функционал (3.3) будет слабо полунепрерывным снизу. В итоге, по лемме 2.3 он будет иметь на $D H$-свойство. 
ЗАмЕчАниЕ 3.3. Если в теореме 3.2 заменить в условии (3.5) член $\sum_{k=0}^{m}|| u_{k}|-| v_{k}||^{p}$ на $\sum_{k=0}^{m}\left|u_{k}-v_{k}\right|^{p}$, то соответствующий функционал (3.3) также будет усиленно выпуклым на $D$, а точнее, строго равномерно выпуклым. Для него остается справедливым результат теоремы 3.2 .

Проиллюстрируем применение теоремы 3.2 на простом примере. Известно, что пространство $W_{p}^{l}(T)$ имеет $H$-свойство при $p>1, l \geqslant 1$, т.е. в нем из слабой сходимости $z_{n} \rightarrow z_{0}$ и сходимости норм $\left\|z_{n}\right\| \rightarrow\left\|z_{0}\right\|$ всегда вытекает сильная сходимость $z_{n} \rightarrow z_{0}$. При $p \geqslant 2$ это следует также и из теоремы 3.2. Действительно, пусть $\Omega(z)=\|z\|_{W_{p}^{l}}^{p}$ и $p \geqslant 2$. Учитывая вид производящей функции $f(x, y)=f(y)=\left|y_{0}\right|^{p}+\left|y_{1}\right|^{p}+\cdots+\left|y_{\nu}\right|^{p}$ этого функционала (см. (3.1)) и используя числовое неравенство

$$
\frac{\left|s_{1}\right|^{p}+\left|s_{2}\right|^{p}}{2} \geqslant\left|\frac{s_{1}+s_{2}}{2}\right|^{p}+\kappa\left|s_{1}-s_{2}\right|^{p} \quad \forall s_{1}, s_{2} \in \mathbb{R}, \quad \kappa=\frac{\min \left\{1,2^{3-p}\right\}}{8}, \quad p \geqslant 2,
$$

получим

$$
\begin{aligned}
\frac{f(u)+f(v)}{2} & =\sum_{k=0}^{\nu} \frac{\left|u_{k}\right|^{p}+\left|v_{k}\right|^{p}}{2} \geqslant \sum_{k=0}^{\nu}\left|\frac{u_{k}+v_{k}}{2}\right|^{p}+\kappa \sum_{k=0}^{\nu}\left|u_{k}-v_{k}\right|^{p} \\
& \geqslant f\left(\frac{u+v}{2}\right)+\left.\kappa \sum_{k=0}^{\nu}|| u_{k}|-| v_{k}\right|^{p} \quad \forall u=\left(u_{0}, \ldots, u_{\nu}\right), \quad v=\left(v_{0}, \ldots, v_{\nu}\right) .
\end{aligned}
$$

Отсюда следует условие (3.5) теоремы 3.2. Эта теорема гарантирует, что усиленно (строго равномерно) вьпукльй при $p \geqslant 2$ функционал $\|z\|_{W_{p}^{l}}^{p}$ имеет $H$-свойство на всем пространстве $W_{p}^{l}(T)$.

Однако условие (3.5) и, в частности, свойство строгой равномерной вьпуклости функционалов, часто не выполнено даже в простейших случаях. Например, тот же функционал $\|z\|_{W_{p}^{l}}^{p}$ при $1<p<2$ уже не будет строго равномерно вьпуклым на всем пространстве $W_{p}^{l}(T)$. Это доказывается как в [4, с. 217] и вытекает из того факта, что неравенства типа (3.6) при $1<p<2$ на всем множестве $\mathbb{R}$ не вьполняются. Как следствие, теорема 3.2 и замечание 3.3 не дают ответа на вопрос об $H$-свойстве нормы в $W_{p}^{l}(T)$ в этом случае. Тем не менее, пространство $W_{p}^{l}(T)$, а, значит, и функционал $\|z\|_{W_{p}^{l}}^{p}$, все же имеет $H$-свойство в случае $1<p<2, l \geqslant 1$.

В оставшейся части этого пункта будут исследоваться классы функционалов общего вида в $W_{p}^{l}(T)$. При этом для более подробного рассмотрения случая $1<p<2$ введем величину

$$
\begin{aligned}
\Delta_{l p}\left(z_{1}, z_{2}\right)= & \sum_{0 \leqslant|\alpha| \leqslant l} \int_{T}\left(\left|D^{\alpha} z_{1}(x)\right|^{2-p}+\left|D^{\alpha} z_{2}(x)\right|^{2-p}\right) \\
& \times\left(\left|D^{\alpha} z_{2}(x)\right|^{p-1}-\left|D^{\alpha} z_{1}(x)\right|^{p-1}\right)^{2} d x \\
= & \sum_{0 \leqslant|\alpha| \leqslant l} \Delta_{p}\left(D^{\alpha} z_{1}(x), D^{\alpha} z_{2}(x)\right) .
\end{aligned}
$$

Изучим ее. 
ЛЕмма 3.4. Функиионал $\Delta_{l p}\left(z_{1}(x), z_{2}(x)\right)$ определен для любых функиий $z_{1}(x)$, $z_{2}(x) \in W_{p}^{l}(T)$ npu $1<p<2$.

ДокАЗАТЕЛЬСтво. Функционал (3.7) состоит из однотипных слагаемых, построенных с помощью величины

$$
\Delta_{p}\left(z_{1}, z_{2}\right)=\int_{T}\left(\left|z_{1}(x)\right|^{2-p}+\left|z_{2}(x)\right|^{2-p}\right)\left(\left|z_{2}(x)\right|^{p-1}-\left|z_{1}(x)\right|^{p-1}\right)^{2} d x,
$$

в которой фигурируют элементы $z_{1}(x), z_{2}(x)$ и $D^{\alpha} z_{1}(x), D^{\alpha} z_{2}(x)$ пространства $L_{p}(T)$. Поэтому достаточно доказать конечность величины $\Delta_{p}\left(z_{1}, z_{2}\right)$ в $L_{p}(T)$. При исследовании на минимум функции

$$
\zeta(t)=\frac{|t|^{p}+1}{2}-\left|\frac{t+1}{2}\right|^{p}-\kappa\left(|t|^{2-p}+1\right)\left(|t|^{p-1}-1\right)^{2}, \quad \kappa=\frac{p(p-1)}{16}, \quad 1<p<2,
$$

оказьвается, что $\zeta(t) \geqslant \zeta_{\min }=\zeta(1)=0$. Приняв $t=s_{1} / s_{2}, s_{1}, s_{2} \in \mathbb{R}$, получим числовое неравенство

$$
\frac{\left|s_{1}\right|^{p}+\left|s_{2}\right|^{p}}{2} \geqslant\left|\frac{s_{1}+s_{2}}{2}\right|^{p}+\kappa\left(\left|s_{1}\right|^{2-p}+\left|s_{2}\right|^{2-p}\right)\left(\left|s_{2}\right|^{p-1}-\left|s_{1}\right|^{p-1}\right)^{2} .
$$

Используя его с заменой $s_{1}, s_{2}$ на функции $z_{1}(x), z_{2}(x) \in L_{p}(T), 1<p<2$, и интегрируя по $x$, получим по свойствам интеграла Лебега

$$
\begin{aligned}
\frac{\left\|z_{1}\right\|_{p}^{p}+\left\|z_{2}\right\|_{p}^{p}}{2} \geqslant & \left\|\frac{z_{1}+z_{2}}{2}\right\|_{p}^{p} \\
& +\kappa \int_{T}\left(\left|z_{1}(x)\right|^{2-p}+\left|z_{2}(x)\right|^{2-p}\right)\left(\left|z_{2}(x)\right|^{p-1}-\left|z_{1}(x)\right|^{p-1}\right)^{2} d x \\
= & \left\|\frac{z_{1}+z_{2}}{2}\right\|_{p}^{p}+\kappa \Delta_{p}\left(z_{1}, z_{2}\right)
\end{aligned}
$$

Отсюда и следует конечность величины $\Delta_{p}\left(z_{1}, z_{2}\right)$.

ЛЕмма 3.5. Пусть $1<p<2$. Предположим, что для последовательности $\left\{z_{n}\right\} \subset L_{p}(T)$ и для әлемента $z_{0} \in L_{p}(T)$ выполнено предельное соотночение $\Delta_{p}\left(z_{n}, z_{0}\right) \rightarrow 0, n \rightarrow \infty$. Тогда имеют место сходимости

$$
\left\|z_{n}\right\|_{p} \rightarrow\left\|z_{0}\right\|_{p},\left.\quad \int_{T}|| z_{n}(x)\right|^{p}-\left|z_{0}(x)\right|^{p} \mid d x \rightarrow 0, \quad n \rightarrow \infty .
$$

ДокАЗАТЕЛЬСтво. Используем известное числовое неравенство

$$
\left.|| s_{2}\right|^{p}-\left|s_{1}\right|^{p}\left|\leqslant \frac{p}{p-1}\left(\left|s_{2}\right|+\left|s_{1}\right|\right)\right|\left|s_{2}\right|^{p-1}-\left|s_{1}\right|^{p-1} \mid \quad \forall s_{1}, s_{2} \in \mathbb{R}, \quad 1<p<2,
$$

для получения оценки

$$
\begin{aligned}
||\left|z_{n}(x)\left\|_{p}^{p}-\right\| z_{0}(x) \|_{p}^{p}\right| & =\left|\int_{T}\left(\left|z_{n}(x)\right|^{p}-\left|z_{0}(x)\right|^{p}\right) d x\right| \leqslant\left.\int_{T}|| z_{n}(x)\right|^{p}-\left|z_{0}(x)\right|^{p} \mid d x \\
& \leqslant\left.\frac{p}{p-1} \int_{T}\left(\left|z_{n}(x)\right|+\left|z_{0}(x)\right|\right)|| z_{n}(x)\right|^{p-1}-\left|z_{0}(x)\right|^{p-1} \mid d x .
\end{aligned}
$$


Применяя в ней справа неравенство Коши-Буняковского, получим

$$
\begin{aligned}
& \left|\left\|z_{n}(x)\right\|_{p}^{p}-\left\|z_{0}(x)\right\|_{p}^{p}\right| \leqslant\left.\int_{T}|| z_{n}(x)\right|^{p}-\left|z_{0}(x)\right|^{p} \mid d x \\
& \leqslant \frac{p}{p-1}\left\{\left[\int_{T}\left|z_{n}(x)\right|^{2(1-p / 2)}\left(\left|z_{n}(x)\right|^{p-1}-\left|z_{0}(x)\right|^{p-1}\right)^{2} d x\right]^{1 / 2}\left[\int_{T}\left|z_{n}(x)\right|^{2(p / 2)} d x\right]^{1 / 2}\right. \\
& \left.\quad+\left[\int_{T}\left|z_{0}(x)\right|^{2(1-p / 2)}\left(\left|z_{n}(x)\right|^{p-1}-\left|z_{0}(x)\right|^{p-1}\right)^{2} d x\right]^{1 / 2}\left[\int_{T}\left|z_{0}(x)\right|^{2(p / 2)} d x\right]^{1 / 2}\right\} \\
& \leqslant \frac{p}{p-1}\left[\int_{T}\left(\left|z_{n}(x)\right|^{2-p}+\left|z_{0}(x)\right|^{2-p}\right)\left(\left|z_{n}(x)\right|^{p-1}-\left|z_{0}(x)\right|^{p-1}\right)^{2} d x\right]^{1 / 2} \\
& \quad \times\left\{\left[\int_{T}\left|z_{n}(x)\right|^{p} d x\right]^{1 / 2}+\left[\int_{T}\left|z_{0}(x)\right|^{p} d x\right]^{1 / 2}\right\} \\
& =\frac{p}{p-1}\left(\left\|z_{n}(x)\right\|_{p}^{p / 2}+\left\|z_{0}(x)\right\|_{p}^{p / 2}\right) \Delta_{p}^{1 / 2}\left(z_{n}, z_{0}\right) .
\end{aligned}
$$

Отсюда и из условия леммы следует, что

$$
\left|\left\|z_{n}(x)\right\|_{p}^{p / 2}-\left\|z_{0}(x)\right\|_{p}^{p / 2}\right| \leqslant \frac{p}{p-1} \Delta_{p}^{1 / 2}\left(z_{n}, z_{0}\right) \rightarrow 0,
$$

т.е., что $\left\|z_{n}\right\|_{p} \rightarrow\left\|z_{0}\right\|_{p}$ при $n \rightarrow \infty$. Тогда из (3.10) и (3.11) получается и другая доказьваемая сходимость:

$$
\left.\int_{T}|| z_{n}(x)\right|^{p}-\left|z_{0}(x)\right|^{p} \mid d x \rightarrow 0
$$

СлЕДСтвиЕ 3.6. Если для последовательности $\left\{z_{n}\right\} \subset W_{p}^{l}(T), 1<p<2$, и для әлемента $z_{0} \in W_{p}^{l}(T)$ выполнено предельное соотношение $\Delta_{l p}\left(z_{n}, z_{0}\right) \rightarrow 0, n \rightarrow \infty$, то справедливо неравенство

$$
\left|\left\|z_{n}\right\|_{W_{p}^{l}}^{p / 2}-\left\|z_{0}\right\|_{W_{p}^{l}}^{p / 2}\right| \leqslant \frac{p \sqrt{\nu}}{p-1} \Delta_{l p}^{1 / 2}\left(z_{n}, z_{0}\right),
$$

из которого следует, что $\left\|z_{n}\right\|_{W_{p}^{l}} \rightarrow\left\|z_{0}\right\|_{W_{p}^{l}}$. Кроме того,

$$
\left.\int_{T}|| z_{n}(x)\right|^{p}-\left|z_{0}(x)\right|^{p} \mid d x \rightarrow 0, \quad n \rightarrow \infty
$$

Действительно, нетрудно убедиться, что вьполнено неравенство

$$
\begin{aligned}
\left|\left\|z_{n}\right\|_{W_{p}^{l}}^{p / 2}-\left\|z_{0}\right\|_{W_{p}^{l}}^{p / 2}\right| & =\left|\sqrt{\sum_{0 \leqslant|\alpha| \leqslant l}\left\|D^{\alpha} z_{n}\right\|_{p}^{p}}-\sqrt{\sum_{0 \leqslant|\alpha| \leqslant l}\left\|D^{\alpha} z_{0}\right\|_{p}^{p}}\right| \\
& \leqslant \sum_{0 \leqslant|\alpha| \leqslant l}\left|\left\|D^{\alpha} z_{n}\right\|_{p}^{p / 2}-\left\|D^{\alpha} z_{0}\right\|_{p}^{p / 2}\right| .
\end{aligned}
$$


Продолжая оценивать его правую часть, применяя при этом аналоги полученной вьше оценки (3.11) и неравенство Коши-Буняковского, получим

$$
\begin{aligned}
\left|\left\|z_{n}\right\|_{W_{p}^{l}}^{p / 2}-\left\|z_{0}\right\|_{W_{p}^{l}}^{p / 2}\right| & \leqslant \frac{p}{p-1} \sum_{0 \leqslant|\alpha| \leqslant l} \Delta_{p}^{1 / 2}\left(D^{\alpha} z_{n}, D^{\alpha} z_{0}\right) \\
& \leqslant \frac{p \sqrt{\nu}}{p-1}\left\{\sum_{0 \leqslant|\alpha| \leqslant l} \Delta_{p}\left(D^{\alpha} z_{n}, D^{\alpha} z_{0}\right)\right\}^{1 / 2}=\frac{p \sqrt{\nu}}{p-1} \Delta_{l p}^{1 / 2}\left(z_{n}, z_{0}\right) \rightarrow 0 .
\end{aligned}
$$

Это доказьвает требуемую сходимость норм при $n \rightarrow \infty$. Вторая доказываемая сходимость получается из леммы 3.5.

ОПРЕДЕЛЕНИЕ 3.7. Функционал общего вида $\Omega$ назьвается квазиравномерно выnуклы.м на вьпуклом множестве $D \subset W_{p}^{l}(T), 1<p<2$, если существует непрерьвная и монотонно возрастающая при $t \geqslant 0$ функция $\xi(t), \xi(0)=0$, такая, что

$$
\frac{\Omega\left(z_{1}\right)+\Omega\left(z_{2}\right)}{2} \geqslant \Omega\left(\frac{z_{1}+z_{2}}{2}\right)+\xi\left[\Delta_{l p}\left(z_{1}, z_{2}\right)\right] \quad \forall z_{1}, z_{2} \in D
$$

Примером квазиравномерно выпуклого функционала на всем пространстве $W_{p}^{l}(T)$, $1<p<2$, будет $\Omega(z)=\|z\|_{W_{p}^{l}}^{p}$. Это устанавливается по той же схеме, что и строгая равномерная вьпуклость нормы при $p \geqslant 2$. Действительно, производящая функция $f(x, y)=f(y)=\left|y_{0}\right|^{p}+\left|y_{1}\right|^{p}+\cdots+\left|y_{\nu}\right|^{p}$ такого функционала в силу неравенства (3.8) обладает свойством

$$
\frac{f(u)+f(v)}{2} \geqslant f\left(\frac{u+v}{2}\right)+\kappa \sum_{k=0}^{\nu}\left(\left|u_{k}\right|^{2-p}+\left|v_{k}\right|^{2-p}\right)\left(\left|u_{k}\right|^{p-1}-\left|v_{k}\right|^{p-1}\right)^{2} \quad \forall u, v \in \mathbb{R}^{\nu+1} .
$$

Отсюда и из определения (3.1) нормы получим условие квазиравномерной вьпуклости c $\xi(t)=\kappa t$ :

$$
\begin{aligned}
& \frac{\left\|z_{1}\right\|_{W_{p}^{l}}^{p}+\left\|z_{2}\right\|_{W_{p}^{l}}^{p}}{2}=\sum_{0 \leqslant|\alpha| \leqslant l} \int_{T} \frac{\left|D^{\alpha} z_{1}(x)\right|^{p}+\left|D^{\alpha} z_{2}(x)\right|^{p}}{2} d x \\
& \geqslant \sum_{0 \leqslant|\alpha| \leqslant l} \int_{T}\left|D^{\alpha}\left(\frac{z_{1}+z_{2}}{2}\right)\right|^{p} d x \\
& \quad+\kappa \sum_{0 \leqslant|\alpha| \leqslant l} \int_{T}\left(\left|D^{\alpha} z_{1}\right|^{2-p}+\left|D^{\alpha} z_{2}\right|^{2-p}\right)\left(\left|D^{\alpha} z_{1}\right|^{p-1}-\left|D^{\alpha} z_{2}\right|^{p-1}\right)^{2} d x \\
& =\left\|\frac{z_{1}+z_{2}}{2}\right\|_{W_{p}^{l}}^{p}+\kappa \Delta_{l p}\left(z_{1}, z_{2}\right) .
\end{aligned}
$$

ТЕОремА 3.8. Если функиионал общего вида $\Omega$ является квазиравномерно выпуклым и слабо полунепрерывным снизу на выпуклом мнохестве $D$, то он обладает на $D$ H-свойством. 
ДокАЗАТЕльство. Квазиравномерная вьпуклость функционала $\Omega$ есть частньй случай усиленной выпуклости (см. определение 2.1$)$ с $\Delta\left(z_{1}, z_{2}\right)=\xi\left[\Delta_{l p}\left(z_{1}, z_{2}\right)\right]$. Пусть для произвольной последовательности $\left\{z_{n}\right\} \subset D$ выполнены соотношения

$$
z_{n} \rightarrow z_{0} \in D, \Delta\left(z_{n}, z_{0}\right) \rightarrow 0, \quad n \rightarrow \infty
$$

Тогда из свойств функции $\xi(t)$ получим

$$
\Delta_{l p}\left(z_{n}, z_{0}\right)=\xi^{-1}\left[\Delta\left(z_{n}, z_{0}\right)\right] \rightarrow 0, \quad n \rightarrow \infty .
$$

Поэтому в силу (3.12) $\left\|z_{n}\right\|_{W_{p}^{l}} \rightarrow\left\|z_{0}\right\|_{W_{p}^{l}}$. Из слабой сходимости последовательности и из доказанной сходимости норм в силу $H$-свойства пространства $W_{p}^{l}(T)$ получается сильная сходимость $z_{n} \rightarrow z_{0}$. Таким образом, функционал $\Delta\left(z_{1}, z_{2}\right)=\xi\left[\Delta_{l p}\left(z_{1}, z_{2}\right)\right]$ имеет по терминологии п. $2 S$-свойство. Но тогда по лемме 2.3 функционал $\Omega$ будет обладать на множестве $D H$-свойством.

Введем более общие классы усиленно выпуклых функционалов, связанных с фиксированными операторами $A_{k} z(x)$.

ОПРЕДЕЛЕНИЕ 3.9. Функционал $\Omega(z)$ называется обобщенно равномерно выпукльм на вьпуклом множестве $D, D \subset W_{p}^{l}(T)$, если существует такая непрерьвная и возрастающая при $t \geqslant 0$ функция $\xi(t), \xi(0)=0$, и непрерывная, возрастающая при $s \geqslant 0$ функция $\varphi(s)$, что для любых $z_{1}, z_{2} \in D$ вьполнено неравенство

$$
\frac{\Omega\left(z_{1}\right)+\Omega\left(z_{2}\right)}{2} \geqslant \Omega\left(\frac{z_{1}+z_{2}}{2}\right)+\xi\left(\Phi\left(z_{1}, z_{2}\right)\right),
$$

где

$$
\Phi\left(z_{1}, z_{2}\right)=\sum_{k=0}^{m} \int_{T}\left|\varphi\left(\left|A_{k} z_{1}\right|\right)-\varphi\left(\left|A_{k} z_{2}\right|\right)\right|^{p} d x .
$$

Функционал $\Omega$ назьвается обобщенно квазиравномерно выпуклым на вьпуклом множестве $D \subset W_{p}^{l}(T), 1<p<2$, если вместо (3.14) выполнено соотношение

$$
\frac{\Omega\left(z_{1}\right)+\Omega\left(z_{2}\right)}{2} \geqslant \Omega\left(\frac{z_{1}+z_{2}}{2}\right)+\xi\left(\Psi\left(z_{1}, z_{2}\right)\right) \quad \forall z_{1}, z_{2} \in D,
$$

в котором

$$
\Psi\left(z_{1}, z_{2}\right)=\sum_{k=0}^{m} \Delta_{p}\left[\varphi\left(\left|A_{k} z_{1}\right|\right), \varphi\left(\left|A_{k} z_{2}\right|\right)\right]
$$

ЗАмЕчАнИЕ 3.10 . Пусть множество $D$ содержит некоторую функцию $\widetilde{z}(x)$, для которой $\varphi\left(\left|A_{k} \widetilde{z}(x)\right|\right) \in L_{p}(T), 0 \leqslant k \leqslant m$. Тогда условия (3.14) или (3.15) порождают включение $\varphi\left(\left|A_{k} z(x)\right|\right) \in L_{p}(T)$ для любой $z(x) \in D$. Действительно, из (3.14) следует

$$
\begin{aligned}
\Phi(z, \widetilde{z}) & =\sum_{k=0}^{m} \int_{T}\left|\varphi\left(\left|A_{k} z\right|\right)-\varphi\left(\left|A_{k} \widetilde{z}\right|\right)\right|^{p} d x \\
& \leqslant \xi^{-1}\left[\frac{\Omega(z)+\Omega(\widetilde{z})}{2}-\Omega\left(\frac{z+\widetilde{z}}{2}\right)\right]<+\infty \quad \forall z(x) \in D \subset W_{p}^{l}(T),
\end{aligned}
$$


т.е. функция $\varphi\left(\left|A_{k} z(x)\right|\right)-\varphi\left(\left|A_{k} \widetilde{z}(x)\right|\right)$, а, значит, и $\varphi\left(\left|A_{k} z(x)\right|\right)$, принадлежит $L_{p}(T)$, $0 \leqslant k \leqslant m$. Если же выполнено (3.15), то по аналогии с неравенством (3.11), заменяя в нем величины $z_{n}(x), z_{0}(x)$ на $\varphi\left(\left|A_{k} z(x)\right|\right), \varphi\left(\left|A_{k} \widetilde{z}(x)\right|\right)$ и проводя оценку как в доказательстве следствия 3.6, получим

$$
\begin{gathered}
\sum_{k=0}^{m}\left|\left\|\varphi\left(\left|A_{k} z\right|\right)\right\|_{p}^{p / 2}-\left\|\varphi\left(\left|A_{k} \widetilde{z}\right|\right)\right\|_{p}^{p / 2}\right| \leqslant \frac{p \sqrt{m}}{p-1}\left\{\sum_{k=0}^{m} \Delta_{p}\left[\varphi\left(\left|A_{k} z_{1}\right|\right), \varphi\left(\left|A_{k} z_{2}\right|\right)\right]\right\}^{1 / 2} \\
=\frac{p \sqrt{m}}{p-1} \Psi^{1 / 2}(z, \widetilde{z}) \leqslant \frac{p \sqrt{m}}{p-1}\left\{\xi^{-1}\left[\frac{\Omega(z)+\Omega(\widetilde{z})}{2}-\Omega\left(\frac{z+\widetilde{z}}{2}\right)\right]\right\}^{1 / 2}<+\infty
\end{gathered}
$$

и это снова доказывает включение $\varphi\left(\left|A_{k} z(x)\right|\right) \in L_{p}(T), 0 \leqslant k \leqslant m$.

ЛЕмма 3.11. Предположим, что для функиии $\varphi(s)$, непрерывной и монотонно возрастающей при $s \geqslant 0$, выполнены неравенства

$$
\varphi(s) \geqslant 0, \quad \varphi(s) \geqslant a+b s \quad \forall s \geqslant 0,
$$

әде $a, b$ - некоторые константы, причем $b>0$. Пусть, далее, последовательность $\left\{z_{n}(x)\right\} \subset D$ и әлемент $z_{0}(x) \in D$ mаковы, что $\varphi\left(\left|z_{n}(x)\right|\right) \in L_{p}(T) u$ $\varphi\left(\left|z_{0}(x)\right|\right) \in L_{p}(T)$. Тогда

1) если имеет место сходимость

$$
\left\|\varphi\left(\left|z_{n}(x)\right|\right)-\varphi\left(\left|z_{0}(x)\right|\right)\right\|_{p} \rightarrow 0 \quad \text { npu } \quad n \rightarrow \infty
$$

$m o\left\|z_{n}\right\|_{p} \rightarrow\left\|z_{0}\right\|_{p}$

2) если при $1<p<2$ справедливо предельное соотношение

$$
\Delta_{p}\left[\varphi\left(\left|z_{n}(x)\right|\right), \varphi\left(\left|z_{0}(x)\right|\right)\right] \rightarrow 0,
$$

mo maкже $\left\|z_{n}\right\|_{p} \rightarrow\left\|z_{0}\right\|_{p}$.

ДокАЗАТЕЛьСтво. В первом случае

$$
\left\|\varphi\left(\left|z_{n}(x)\right|\right)-\varphi\left(\left|z_{0}(x)\right|\right)\right\|_{p} \rightarrow 0
$$

т.е.

$$
v_{n}(x)=\left|v_{n}(x)\right|=\varphi\left(\left|z_{n}(x)\right|\right) \stackrel{L_{p}}{\rightarrow} \varphi\left(\left|z_{0}(x)\right|\right)=\left|v_{0}(x)\right|=v_{0}(x) \in L_{p}(T) .
$$

Исследуем предельное соотношение между функциями $\left|z_{n}(x)\right|=\varphi^{-1}\left(\left|v_{n}(x)\right|\right)$ и $\left|z_{0}(x)\right|=$ $\varphi^{-1}\left(\left|v_{0}(x)\right|\right)$ при $n \rightarrow \infty$. По условиям на функцию $v=\varphi(s)$ получим

$$
0 \leqslant s=\varphi^{-1}(v)=\varphi^{-1}(|v|) \leqslant a_{0}+b_{0} v=a_{0}+b_{0}|v|
$$

где $a_{0}=-a / b, b_{0}=1 / b$. Таким образом, $\left|\varphi^{-1}(|v|)\right| \leqslant a_{0}+b_{0}|v|$. Это условие согласно результатам из [5, с. 204], [6, с. 312] гарантирует, что оператор $\varphi^{-1}[\cdot]$, которьй действует по правилу $|z(x)|=\varphi^{-1}[|v(x)|]$ на элементы $v(x)$ пространства $L_{p}(T)$, будет непрерьвньм из $L_{p}$ в $L_{p}$. Следовательно,

$$
\left|z_{n}(x)\right|=\varphi^{-1}\left[\left|v_{n}(x)\right|\right] \stackrel{L_{p}}{\rightarrow} \varphi^{-1}\left[\left|v_{0}(x)\right|\right]=\left|z_{0}(x)\right|,
$$


откуда и получается сходимость норм $\left\|z_{n}\right\|_{p} \rightarrow\left\|z_{0}\right\|_{p}$. Во втором случае, применяя лемму 3.5 с заменой в ней функций $z_{n}=z_{n}(x), z_{0}=z_{0}(x)$ на $\varphi\left(\left|z_{n}(x)\right|\right), \varphi\left(\left|z_{0}(x)\right|\right)$ соответственно и учитывая условие $\varphi(s) \geqslant 0$, получим

$$
\int_{T}\left|\varphi^{p}\left(\left|z_{n}(x)\right|\right)-\varphi^{p}\left(\left|z_{0}(x)\right|\right)\right| d x \rightarrow 0 \quad \text { при } n \rightarrow \infty .
$$

Это значит, что

$$
w_{n}(x)=\varphi^{p}\left(\left|z_{n}\right|\right) \stackrel{L_{1}}{\rightarrow} \varphi^{p}\left(\left|z_{0}\right|\right)=w_{0}(x) \in L_{1}(T) .
$$

Используем далее тот факт, что оператор возведения в степень $v(x)=|w(x)|^{1 / p}$ непрерьвен из $L_{1}$ в $L_{p}$, как это следует из [5, с. 204], [6, с. 312]. Поэтому

$$
v_{n}(x)=\varphi\left(\left|z_{n}(x)\right|\right)=\left|\varphi^{p}\left(\left|z_{n}(x)\right|\right)\right|^{1 / p} \stackrel{L_{p}}{\rightarrow}\left|\varphi^{p}\left(\left|z_{0}(x)\right|\right)\right|^{1 / p}=\varphi\left(\left|z_{0}(x)\right|\right)=v_{0}(x) .
$$

Дальнейшие рассуждения, использующие условия $\varphi(s) \geqslant 0, \varphi(s) \geqslant a+b s$, проводятся аналогично доказательству первой части этой леммы и снова дают сходимость $\left\|z_{n}\right\|_{p} \rightarrow\left\|z_{0}\right\|_{p}$

ТЕОРемА 3.12. Предположим, что для функиии $\varphi(s)$, включенной в определение 3.9 , выполнены условия

$$
\varphi(s) \geqslant 0, \quad \varphi(s) \geqslant a+b s \quad \forall s \geqslant 0, \quad a, b=\text { const, } \quad b>0 .
$$

Пусть, далее, выпуклое мнохество $D, D \subset W_{p}^{l}(T)$, содержит такую функиию $\widetilde{z}(x)$, что $\varphi\left(\left|A_{k} \widetilde{z}\right|\right) \in L_{p}(T)$ при всех $k=0, \ldots, m$ (например, $\widetilde{z}(x)=C_{0}=$ const $\in D)$, а функиионал $\Omega(z)$ слабо полунепрерывен снизу на $D$. Тогда

1) если функиионал $\Omega(z)$ является обобщенно равномерно выпуклым на множестве $D$, то он обладает $H$-свойством;

2) если же функиионал $\Omega(z)$ обобщенно квазиравномерно выпуклый на выпуклом мнохсестве $D \subset W_{p}^{l}(T), 1<p<2$, то он также имеет $H$-свойство.

ДокАЗАТЕЛЬСТво. Обобщенная равномерная вьпуклость есть вариант усиленной выпуклости функционала $\Omega(z)$ с $\Delta\left(z_{1}, z_{2}\right)=\xi\left[\Phi\left(z_{1}, z_{2}\right)\right]$. Возьмем произвольную последовательность $\left\{z_{n}\right\} \subset D$ со свойствами $z_{n} \rightarrow z_{0} \in D$ и $\xi\left[\Phi\left(z_{n}, z_{0}\right)\right] \rightarrow 0$ при $n \rightarrow \infty$. Тогда

$$
\sum_{k=0}^{m} \int_{T}\left|\varphi\left(\left|A_{k} z_{n}\right|\right)-\varphi\left(\left|A_{k} z_{0}\right|\right)\right|^{p} d x \rightarrow 0
$$

и, значит, по лемме 3.11 (с учетом замечания 3.10 )

$$
\left\|A_{k} z_{n}\right\|_{p} \rightarrow\left\|A_{k} z_{0}\right\|_{p} \quad \forall k \quad \text { при } n \rightarrow \infty .
$$

Далее, повторяя буквально соответствующую часть доказательства теоремы 3.2 , получим из этой сходимости норм, что $z_{n} \rightarrow z_{0}$ в $W_{p}^{l}(T)$, и это означает вьполнение $S$-свойства для функционала $\Delta\left(z_{1}, z_{2}\right)=\xi\left[\Phi\left(z_{1}, z_{2}\right)\right]$. По лемме 2.3 обобщенно равномерно выпукльй и слабо полунепрерьвньй снизу на $D$ функционал $\Omega(z)$ будет тогда обладать $H$-свойством. Пусть теперь $\Omega(z)$ - обобщенно квазиравномерно выпукльй на множестве $D \subset W_{p}^{l}(T), 1<p<2$, функционал. Тогда он усиленно выпукльй с $\Delta\left(z_{1}, z_{2}\right)=\xi\left[\Psi\left(z_{1}, z_{2}\right)\right]$. Выбрав снова произвольную последовательность, для которой $z_{n} \rightarrow z_{0} \in D$ и $\xi\left[\Psi\left(z_{n}, z_{0}\right)\right] \rightarrow 0$, получим $\Psi\left(z_{n}, z_{0}\right) \rightarrow 0$, т.е. $\Delta_{p}\left[\varphi\left(\left|A_{k} z_{n}\right|\right), \varphi\left(\left|A_{k} z_{0}\right|\right)\right]$ $\rightarrow 0$. Тогда по лемме $3.11\left\|A_{k} z_{n}\right\|_{p} \rightarrow\left\|A_{k} z_{0}\right\|_{p}$ для всех $k$. Дальнейшее доказательство проводится как для случая обобщенной равномерной выпуклости. 
4. Достаточные условия усиленной выпуклости функционалов в пространствах Соболева. Обратимся снова к функционалам вида (3.3). Для того чтобы они были обобщенно равномерно выпуклы или обобщенно квазиравномерно вьпуклы на вьпуклом множестве $D \subset W_{p}^{l}(T)$, достаточно, например, потребовать для производящей функции $f(x, y)=f\left(x, y_{0}, y_{1}, \ldots, y_{m}\right)$ вьполнение условий а) и б), а также выполнения одного из следующих неравенств:

$$
\begin{gathered}
\frac{f(x,|u|)+f(x,|v|)}{2} \geqslant f\left(x,\left|\frac{u+v}{2}\right|\right)+\kappa \sum_{k=0}^{m}\left|\varphi\left(\left|u_{k}\right|\right)-\varphi\left(\left|v_{k}\right|\right)\right|^{p} \\
\forall x \in T, \quad \forall u, v \in \mathbb{R}^{m+1}, \quad \kappa=\mathrm{const}>0, \quad p>1, \\
\frac{f(x,|u|)+f(x,|v|)}{2} \geqslant f\left(x,\left|\frac{u+v}{2}\right|\right)+\kappa \sum_{k=0}^{m}\left(\varphi^{2-p}\left(\left|u_{k}\right|\right)+\varphi^{2-p}\left(\left|v_{k}\right|\right)\right) \\
\quad \times\left(\varphi^{p-1}\left(\left|u_{k}\right|\right)-\varphi^{p-1}\left(\left|v_{k}\right|\right)\right)^{2}, \quad 1<p<2,
\end{gathered}
$$

с функцией $\varphi(s)$, которая удовлетворяет требованиям из определения 3.9 и условиям леммы 3.11 (теоремы 3.12). Тогда, с одной стороны, функция $f(x, y)$ будет подчиняться условиям а)-в) и, значит, по лемме 3.1 соответствующий функционал (3.3) будет слабо полунепрерьвньм снизу. С другой стороны, для такого функционала (3.3) будет вьполнено неравенство (3.14) или (3.15) с $\xi(t)=\kappa t$. В этом нетрудно убедиться по аналогии с доказательством теоремы 3.2 с заменой там условия (3.5) на (4.1) или (4.2). Значит, по теореме 3.12 такой функционал (3.3) будет иметь $H$-свойство. При $\varphi(s)=s$ неравенства (4.1) и (4.2) аналогично обеспечивают строгую равномерную и квазиравномерную выпуклость функционала (3.3) соответственно и по теоремам $3.2,3.8$ - его $H$-свойство.

Интерес, однако, представляют более детальные достаточные условия на производящие функции для выполнения $H$-свойства функционалов, чем просто неравенства (4.1), (4.2). Такие условия можно получить для функционалов (3.3) специального вида

$$
\widehat{\Omega}(z)=\int_{T} f\left(x, \varphi^{p}(|z(x)|), \varphi^{p}\left(\left|A_{1} z(x)\right|\right), \ldots, \varphi^{p}\left(\left|A_{m} z(x)\right|\right)\right) d x
$$

определенных на выпуклом множестве $D \subset W_{p}^{l}(T)$.

ТЕорема 4.1. Предположим, что

1) функиия $f(x, y) \geqslant 0$ определена при $x \in T, y=\left(y_{0}, y_{1}, \ldots, y_{m}\right) \in \mathbb{R}_{+}^{m+1} u$ непрерывна там;

2) она дифферениируема по у при каждом $x \in T$, причем $f_{y_{i}}^{\prime}(x, y) \geqslant \gamma=$ const $>0$, $i=0,1, \ldots, m, \forall(x, y) \in T \times \mathbb{R}_{+}^{m+1}$

3) она выпукла по у в $\mathbb{R}_{+}^{m+1}$ при каждом $x \in T$;

4) функиия $\varphi(s)$ непрерывна, неотрицательна, монотонно возрастает и выпукла при $s \geqslant 0$.

Тогда функиионал (4.3) является обобщенно квазиравномерно выпуклым при $1<$ $p<2$ и обобщенно равномерно выпуклым при $p \geqslant 2$ на D.

ДокАЗАТЕльСтво. Пусть, например, $p \geqslant 2$. Тогда, используя вьпуклость функции $f(x, y)$ по переменным $y$, монотонность функции $f(x, y)=f\left(x, y_{0}, y_{1}, \ldots, y_{m}\right)$ по аргументам $y_{k}, k=0,1, \ldots, m$, вытекающую из условия 2$)$, применяя неравенство типа (3.6) с заменой в нем чисел $s_{1}, s_{2}$ на $\varphi^{p}\left(\left|u_{k}\right|\right)$ и $\varphi^{p}\left(\left|v_{k}\right|\right), k=0,1, \ldots, m$, обозначая 
$\varphi^{p}(|u|)=\left(\varphi^{p}\left(\left|u_{0}\right|\right), \ldots, \varphi^{p}\left(\left|u_{m}\right|\right)\right)$ и используя формулу конечных приращений, получим

$$
\begin{aligned}
& \frac{f\left(x, \varphi^{p}(|u|)\right)+f\left(x, \varphi^{p}(|v|)\right)}{2} \geqslant f\left(x, \frac{\varphi^{p}(|u|)+\varphi^{p}(|v|)}{2}\right) \\
& \geqslant f\left(x,\left|\frac{\varphi(|u|)+\varphi(|v|)}{2}\right|^{p}+\kappa|\varphi(|u|)-\varphi(|v|)|^{p}\right) \\
&= f\left(x,\left|\frac{\varphi(|u|)+\varphi(|v|)}{2}\right|^{p}\right) \\
&+\sum_{k=0}^{m} f_{y_{k}}^{\prime}\left(x,\left|\frac{\varphi(|u|)+\varphi(|v|)}{2}\right|^{p}+\kappa \theta|\varphi(|u|)-\varphi(|v|)|^{p}\right) \cdot \kappa\left|\varphi\left(\left|u_{k}\right|\right)-\varphi\left(\left|v_{k}\right|\right)\right|^{p} \\
& \geqslant f\left(x,\left|\frac{\varphi(|u|)+\varphi(|v|)}{2}\right|^{p}\right)+\gamma \kappa \sum_{k=0}^{m}\left|\varphi\left(\left|u_{k}\right|\right)-\varphi\left(\left|v_{k}\right|\right)\right|^{p} \\
& \forall u=\left(u_{0}, u_{1}, \ldots, u_{m}\right), \quad v=\left(v_{0}, v_{1}, \ldots, v_{m}\right), \quad 0<\theta<1 .
\end{aligned}
$$

Продолжим эту вькладку с учетом возрастания функции $f(x, y)$ по аргументам $y$, выпуклости функции $\varphi(s) \geqslant 0$ и ее возрастания при $s \geqslant 0$ :

$$
f\left(x,\left|\frac{\varphi(|u|)+\varphi(|v|)}{2}\right|^{p}\right) \geqslant f\left(x, \varphi^{p}\left(\frac{|u|+|v|}{2}\right)\right) \geqslant f\left(x, \varphi^{p}\left(\left|\frac{u+v}{2}\right|\right)\right) .
$$

В итоге, для каждого $x \in T$ и любых $u, v \in \mathbb{R}^{m+1}$ получим

$$
\frac{f\left(x, \varphi^{p}(|u|)\right)+f\left(x, \varphi^{p}(|v|)\right)}{2} \geqslant f\left(x, \varphi^{p}\left(\left|\frac{u+v}{2}\right|\right)\right)+\gamma \kappa \sum_{k=0}^{m}\left|\varphi\left(\left|u_{k}\right|\right)-\varphi\left(\left|v_{k}\right|\right)\right|^{p} .
$$

Подставляя в это числовое неравенство вместо $u_{k}, v_{k}$ величины $A_{k} z_{1}(x), A_{k} z_{2}(x)$ для произвольных функций $z_{1}(x), z_{2}(x)$ из вьпуклого множества $D \subset W_{p}^{l}(T)$ и почленно интегрируя полученное соотношение, приходим к следуюшему варианту формулы (3.14):

$$
\begin{gathered}
\frac{\widehat{\Omega}\left(z_{1}\right)+\widehat{\Omega}\left(z_{2}\right)}{2} \geqslant \widehat{\Omega}\left(\frac{z_{1}+z_{2}}{2}\right)+\gamma \kappa \sum_{k=0}^{m} \int_{T}\left|\varphi\left(\left|A_{k} z_{1}(x)\right|\right)-\varphi\left(\left|A_{k} z_{2}(x)\right|\right)\right|^{p} d x \\
\forall z_{1}, z_{2} \in D .
\end{gathered}
$$

Это доказьвает обобщенную равномерную вьпуклость функционала (4.3).

В случае $1<p<2$ обобщенная квазиравномерная вьпуклость доказьвается по той же схеме с некоторыми изменениями. Так, вместо неравенства (3.6) используется неравенство (3.8) с заменой в нем чисел $s_{1}, s_{2}$ на $\varphi\left(\left|u_{k}\right|\right), \varphi\left(\left|v_{k}\right|\right)$ соответственно и с последующей заменой в выкладках члена $\left|\varphi\left(\left|u_{k}\right|\right)-\varphi\left(\left|v_{k}\right|\right)\right|^{p}$ на

$$
\left(\varphi^{2-p}\left(\left|u_{k}\right|\right)+\varphi^{2-p}\left(\left|v_{k}\right|\right)\right)\left(\varphi^{p-1}\left(\left|u_{k}\right|\right)-\varphi^{p-1}\left(\left|v_{k}\right|\right)\right)^{2} .
$$

В частности, так модифицируется неравенство (4.4). В итоге получается вариант соотношения (3.15):

$$
\frac{\widehat{\Omega}\left(z_{1}\right)+\widehat{\Omega}\left(z_{2}\right)}{2} \geqslant \widehat{\Omega}\left(\frac{z_{1}+z_{2}}{2}\right)+\gamma \kappa \sum_{k=0}^{m} \Delta_{p}\left[\varphi\left(\left|A_{k} z_{1}\right|\right), \varphi\left(\left|A_{k} z_{2}\right|\right)\right] \quad \forall z_{1}, z_{2} \in D,
$$

и это доказывает теорему. 
СлЕДСТВИЕ 4.2. Если множество D удовлетворяет условию теоремы 3.12 (например, содержит некоторую постоянную функиию), а для функиий $f u \varphi$ выполнены условия теоремы 4.1 и, кроме того, как и в теореме 3.12, условие $\varphi(s) \geqslant a+b s(b>0)$ при $s \geqslant 0$, то функиионал (4.3) слабо полунепрерывен снизу на $D$ и обладает там $H$-свойством.

Действительно, производящая функция $f_{0}(x, y)=f\left(x, \varphi^{p}(y)\right)$ функционала (4.3) имеет свойства а)-в) из п. 3. Еенепрерьвность и монотонность следует из условий 1), 2) и 4) теоремы 4.1, а ее выпуклость вытекает из неравенства (4.4) при $p \geqslant 2$ или его аналога при $1<p<2$. Поэтому для доказательства остается применить лемму 3.1 , теорему 4.1 и теорему 3.12 .

Рассмотрим конкретные примеры. Как нетрудно убедиться, следующие функционалы с $\varphi(s)$, подчиненной условиям теоремы 3.12 , оказьваются по теореме 4.1 обобщенно равномерно выпуклыми при $p \geqslant 2$ и обобщенно квазиравномерно вьпуклыми при $1<p<2$ на $D$ :

$$
\begin{aligned}
& \Omega_{1}(z)=\sum_{k=0}^{m} \int_{T} q(x) \exp \left[\varphi^{p}\left(\left|A_{k} z(x)\right|\right)\right] d x, \\
& \Omega_{2}(z)=\sum_{k=0}^{m} \int_{T} q(x)\left[\varphi^{p}\left(\left|A_{k} z(x)\right|\right)+1\right] \ln \left[\varphi^{p}\left(\left|A_{k} z(x)\right|\right)+1\right] d x .
\end{aligned}
$$

Здесь $q(x) \geqslant q_{0}=$ const $>0$ - заданная непрерьвная в $T$ функция. По следствию 4.2 функционалы $\Omega_{1,2}(z)$, будучи слабо полунепрерьвньми снизу, имеют $H$-свойство на $D$.

Особый интерес представляет функционал

$$
\Omega_{3}(z)=\sum_{k=0}^{m} \int_{T} q(x)\left[\left|A_{k} z(x)\right|+r\right]^{p} \ln ^{p}\left[\frac{\left|A_{k} z(x)\right|+r}{r}\right] d x
$$

определенный на некотором вьпуклом множестве $D \subset W_{p}^{l}(T)$. Здесь $r>0$ - заданная константа. При $Z=L_{1}(T)$ близкий по виду функционал

$$
\Omega_{E}(z)=\int_{T} z(x) \ln \frac{z(x)}{r} d x,
$$

определенный на $D_{r}=\left\{z(x) \in L_{1}(T): z(x) \geqslant r, \Omega_{E}(z)<\infty\right\}$ и используемый в так назьваемом методе максимальной энтропии, хорошо изучен (см., например, [7], [8]). В частности, в [3] фактически установлено его $H$-свойство на $D_{r}$. Функционал $(4.5)$ с этой точки зрения ранее не изучался. Производящая функция функционала (4.5)

$$
f(x, y)=q(x) \sum_{k=0}^{m} y_{k}
$$

удовлетворяет на множестве $T \times \mathbb{R}^{m+1}$ условиям теоремы 4.1. Условиям этой теоремы подчинена и функция

$$
\varphi(s)=(s+r) \ln \left(\frac{s+r}{r}\right), \quad s \geqslant 0 .
$$

Кроме того, $\varphi(0)=0, \varphi(s) \geqslant s$. Поэтому по теореме 4.1 функционал $\Omega_{3}(z)$ будет обобщенно равномерно вьпуклым при $p \geqslant 2$ или обобщенно квазиравномерно вьпуклым при 
$1<p<2$ на множестве $D$. По следствию 4.2 он будет слабо полунепрерьвным снизу на $D$ и будет иметь там $H$-свойство.

Теперь рассмотрим часто встречающиеся в приложениях функционалы вида

$$
\Omega(z)=\sum_{k=0}^{m} \int_{T} q(x)\left|A_{k} z(x)\right|^{p} \psi\left(\left|A_{k} z(x)\right|\right) d x, \quad z(x) \in D \subset W_{p}^{l}(T),
$$

где $q(x), \psi(z)$ - некоторые заданные функции.

Теорема 4.3. Предположим, что $q(x) \geqslant q_{0}=$ const $>0$ - непрерывная в $T$ функиия, а неотрицательная и непрерывная при $s \geqslant 0$ функция $\psi(s)$ такова, что при рассматриваемом числе $p$ функция $\omega(s)=(\psi(|s|)-1)|s|^{p}$ выпукла. Тогда функиионал (4.6) будет строго равномерно выпуклым при $p \geqslant 2$ и квазиравномерно выпуклым при $1<p<2$ на выпуклом множестве $D$.

ДокАЗАТЕЛЬСТво. Положим $f\left(x, y_{k}\right)=q(x) y_{k}^{p} \psi\left(y_{k}\right), y_{k} \geqslant 0$, и пусть, например, $1<p<2$. Тогда для любых $u_{k}, v_{k} \in \mathbb{R}$ из неравенства (3.8), а также из неравенства $q(x) \geqslant q_{0}$ и выпуклости функции $\omega(s)=(\psi(|s|)-1)|s|^{p}$, следует оценка

$$
\begin{aligned}
\frac{f\left(x,\left|u_{k}\right|\right)+f\left(x,\left|v_{k}\right|\right)}{2}=q(x) \frac{\left|u_{k}\right|^{p}+\left|v_{k}\right|^{p}+\left|u_{k}\right|^{p}\left(\psi\left(\left|u_{k}\right|\right)-1\right)+\left|v_{k}\right|^{p}\left(\psi\left(\left|v_{k}\right|\right)-1\right)}{2} \\
\geqslant q(x)\left|\frac{u_{k}+v_{k}}{2}\right|^{p}+q_{0} \kappa\left(\left|u_{k}\right|^{2-p}+\left|v_{k}\right|^{2-p}\right)\left(\left|u_{k}\right|^{p-1}-\left|v_{k}\right|^{p-1}\right)^{2} \\
\quad+q(x)\left|\frac{u_{k}+v_{k}}{2}\right|^{p}\left(\psi\left(\left|\frac{u_{k}+v_{k}}{2}\right|\right)-1\right) \\
=q(x)\left|\frac{u_{k}+v_{k}}{2}\right|^{p} \psi\left(\left|\frac{u_{k}+v_{k}}{2}\right|\right)+q_{0} \kappa\left(\left|u_{k}\right|^{2-p}+\left|v_{k}\right|^{2-p}\right)\left(\left|u_{k}\right|^{p-1}-\left|v_{k}\right|^{p-1}\right)^{2} \\
=f\left(x,\left|\frac{u_{k}+v_{k}}{2}\right|\right)+q_{0} \kappa\left(\left|u_{k}\right|^{2-p}+\left|v_{k}\right|^{2-p}\right)\left(\left|u_{k}\right|^{p-1}-\left|v_{k}\right|^{p-1}\right)^{2} .
\end{aligned}
$$

Отсюда ясно, что выполнено соотношение $(4.2)$ с $\varphi(s)=s$, из которого, как это указано в начале п. 4 , получается условие квазиравномерной выпуклости (3.13) функционала (4.6) на выпуклом множестве $D$ c $\xi(t)=q_{0} \kappa t$. Эта теорема доказьвается аналогично и для случая $p \geqslant 2$, если вместо (3.8) использовать неравенство (3.6). Соответственно меняется выкладка (4.7), и вместо (4.2) получается вариант соотношения (4.1).

СледСТВИЕ 4.4. Если функиии q, $\psi$ подчинены условиям теоремы 4.3 и, кроме того, $\psi(s)$ не убывает при $s \geqslant 0$, то производящая функиия

$$
f(x, y)=q(x) \sum_{k=0}^{m} y_{k}^{p} \psi\left(y_{k}\right)
$$

функиионала (4.6) имеет при $y \in \mathbb{R}_{+}^{m+1}, x \in T$ свойства а)-в). Тогда по лемме 3.1 функиионал (4.6) полунепрерывен снизу, а по теореме 3.2 (при $p \geqslant 2$ ) или по теореме 3.8 (при $1<p<2$ ) с учетом результата теоремы 4.3 он имеет $H$-свойство на $D$.

В качестве конкретного примера таких функционалов (4.6) можно указать

$$
\Omega_{4}(z)=\sum_{k=0}^{m} \int_{T} q(x)\left|A_{k} z(x)\right|^{p} \exp \left(\left|A_{k} z(x)\right|\right) d x
$$


и ряд других.

В заключении отметим, что утверждения об $H$-свойстве могут быть по близкой методике доказаны и для аналогов функционалов $(3.3),(4.3)$ и (4.6)

$$
\begin{gathered}
\bar{\Omega}(z)=\int_{T} f(x,|z(x)|) d x, \quad \widehat{\Omega}(z)=\int_{T} f\left(x, \varphi^{p}(|z(x)|)\right) d x \\
\Omega(z)=\int_{T} q(x)|z(x)|^{p} \psi(|z(x)|) d x
\end{gathered}
$$

в пространстве $L_{p}(T), p>1$.

\section{СПИСОК ЦИТИРОВАННОЙ ЛИТЕРАТУРЫ}

[1] Diestel J. Geometry of Banach Spaces—Selected Topics. № 11. Lecture Notes in Math. V. 485. Berlin-Heidelberg: Springer-Verlag, 1975.

[2] Канторович Л.В., Акилов Г. П. Функциональный анализ. М.: Наука, 1977.

[3] Леонов А.С. Обобщение метода максимальной энтропии для решения некорректных задач // Сиб. матем. ж. 2000. Т. 41. № 4. С. 863-872.

[4] Васильев Ф. П. Численные методы решения экстремальных задач. М.: Наука, 1980.

[5] Вайнберг М. М. Вариационные методы исследования нелинейных операторов. М.: ГИТТЛ, 1956.

[6] Крейн С.Г. (ред. ) Функциональньй анализ (Серия СМБ). М.: Наука, 1972.

[7] Amato U., Hughes W. Maximum entropy regularization of Fredholm integral equations of the first kind // Inverse Problems. 1991. V. 7. P. 793-808.

[8] Eggermont P. P. B. Maximum entropy regularization for Fredholm integral equations of the first kind // SIAM J. Math. Anal. 1993. V. 24. №6. P. 1557-1576.

Московский инженерно-физический институт (технический университет)

Поступило

E-mail : leonov@illposed.msk.ru

27.06 .2002 